\title{
The anti-atherosclerosis effect of anakinra, a recombinant human interleukin-1 receptor antagonist, in apolipoprotein E knockout mice
}

Eu Jeong Ku ( $\nabla$ eujeong.ku@gmail.com )

Chungbuk National University Hospital; Chungbuk National University College of Medicine https://orcid.org/0000-0001-5533-4989

\section{Bo-Rahm Kim}

Seoul National University Bundang Hospital Department of Internal Medicine Jee-In Lee

Seoul National University Bundang Hospital Department of Internal Medicine

\section{Yun-Kyung Lee}

Seoul National University Bundang Hospital Department of Internal Medicine

\section{Tae Jung Oh}

Seoul National University Bundang Hospital Department of Internal Medicine

Hak C. Jang

Seoul National University Bundang Hospital Department of Internal Medicine

\section{Sung Hee Choi}

Seoul National University Bundang Hospital Department of Internal Medicine

\section{Research Article}

Keywords: Atherosclerosis, IL-1 receptor blocker, Anakinra, Smooth muscle cell migration, Antiinflammation

Posted Date: June 12th, 2021

DOl: https://doi.org/10.21203/rs.3.rs-482370/v1

License: (c) (i) This work is licensed under a Creative Commons Attribution 4.0 International License. Read Full License 


\section{Abstract}

Purpose Interleukin (IL)-1 $\beta$ plays an important role in the atherosclerosis pathogenesis. We aimed to investigate the effect of anakinra, a recombinant human IL-1 receptor antagonist, on the progression of atherosclerosis in apolipoprotein $\mathrm{E}$ knockout $\left(\mathrm{ApoE}^{-/-}\right)$mice.

Methods ApoE ${ }^{-/-}$mice (8-week male) were treated with saline (control), anakinra 10, 25, and $50 \mathrm{mg} / \mathrm{kg}$, respectively ( $n=10$ in each group). Mice were fed a standard chow ( 4 weeks) followed by an atherogenic diet (35kcal\% fat, $1.25 \%$ cholesterol, 12 weeks). Atheromatous plaques and expression of inflammatory genes and signaling pathways in human umbilical vein endothelial cells (HUVECs), rat aortic smooth muscle cells (RAOSMCs), and 3T3-L1 adipocytes were assessed.

Results The plaque size of aortic arch $(30.6 \%$ and $25.2 \%$ at the $25 \mathrm{mg} / \mathrm{kg}$ and $50 \mathrm{mg} / \mathrm{kg}$ doses, both $P<$ 0.05 ) and serum triglyceride were significantly reduced in anakinra-treated $\mathrm{ApoE}^{-/-}$mice and inflammatory genes (IL-1 $\beta$ and IL-6) expressions in anakinra-treated HUVECs and RAOSMCs were suppressed (all $P<0.05$ ). In RAOSMCs, anakinra reduced metalloproteinase-9 expression in a dosedependent manner and inhibited RAOSMC migration in a wound healing assay. Anakinra-treated mice exhibited a trend toward lower CD68 + macrophage infiltration in visceral fat and monocyte chemoattractant protein-1 expression was reduced in 3T3-L1 adipocytes.

Conclusion IL-1 blockade with anakinra significantly reduced atherosclerotic plaque formation in $\mathrm{ApoE}^{-/-}$ mice. Also, anakinra reduced expression of inflammatory genes in endothelial cells, smooth muscle cells, and adipocytes and ameliorated processes involved in vascular remodeling. Anakinra could be a useful component of complementary treatment with standard regimen to reduce the residual cardiovascular risk.

\section{Introduction}

Atherosclerotic cardiovascular diseases (CVDs) are the major cause of mortality worldwide [1]. There is widespread evidence to support the use of high-intensity statin treatment to reduce low-density lipoprotein cholesterol (LDL-C) effectively as the first-line treatment for atherosclerosis. However, the residual risk for developing cardiovascular events after statin treatment remains up to $50-60 \%$. The accumulating body of evidence in humans and animals suggests that chronic inflammation plays a critical role in the process of atherosclerosis [2]. Beyond the roles of lipid-lowering therapy with statins, ezetimibe, and pro-protein convertase subtilisin/kexin type 9 inhibitor, the effective inhibition of chronic inflammation may be an important component of anti-atherosclerosis treatment.

Interleukin (IL)-1 $\beta$ is an important mediator of inflammatory responses, driving the expression of mediators such as cyclooxygenase-2, IL-1, IL-6, IL-12, intercellular adhesion molecule-1 (ICAM-1), vascular cell adhesion molecule-1, and the tumor necrosis factor-a (TNF-a) signaling pathway, which all contribute to the development of vascular remodeling and atherosclerosis [3-6]. The inflammasome is an 
intracellular multiprotein complex that activates a pro-inflammatory cascade in response to signals from microbe-derived pathogen-associated molecular patterns and host cell-generated danger-associated molecular patterns [7]. Notably, intracellular protein NACHT, LRR, and PYD domain-containing protein 3 (NLRP3) forms the NLRP3 inflammasome, which mediates the effects of IL-1 $\beta$ related to atherosclerosis. This process causes the induction of expression of various inflammatory cytokines and chemokines, the increased expression of leukocyte adhesion molecules in endothelial cells, the activation of cell proliferation, remodeling, and migration molecules in smooth muscle cells, and the alteration of monocytes/macrophages involved in innate immunity [8].

Based on these findings, several trials have been conducted with the aim of applying anti-inflammatory therapy targeting the ligands or receptors for the IL-1 family to treat CVD [[9], [10], [11]]. Anti-IL-1 agents have shown particularly promising results in basic and translational studies, and further, have been reported to have effects on CVD including acute myocardial (MI) infarction, heart failure, and pericarditis [12-14].

Anakinra, a recombinant human IL-1 receptor antagonist that blocks the biological cascades of IL-1, has applications in the reduction of systemic inflammatory responses. In 2001, it was approved by the US Food and Drug Administration for the treatment of rheumatoid arthritis, and has shown significant therapeutic effects on a range of systemic autoimmune diseases such as cryopyrin-associated periodic syndromes and juvenile and adult onset Still's disease [15]. Several small clinical trials using anakinra as a putative treatment strategy for CVD rather than systemic autoimmune diseases have been reported [16-18]. In these previous studies, anakinra produced relatively short-term changes in inflammatory biomarkers, or improvement of cardiac function. However, the use of anakinra to treat atherosclerosis needs more validation and requires investigation of the mechanistic role of anakinra in the progression of atherosclerotic CVD.

This study aimed to investigate the anti-atherosclerosis effects of chronic (16 weeks) treatment with anakinra to block IL-1 activity in apolipoprotein $\mathrm{E}$ knockout $\left(\mathrm{ApoE}^{-/-}\right)$mice fed an atherogenic diet, and to explore its possible mechanisms of action.

\section{Methods}

\section{Animals, diet, and treatment protocols}

All experiment protocols were approved by the Seoul National University Bundang Hospital Institutional Animal Care and Use Committee (BA1511-188/070 - 01). Animal experiments were performed in compliance with the guidelines from Directive 2010/63/EU of the European Parliament on the protection of animals used for scientific purposes or the NIH Guide for the Care and Use of Laboratory Animals.

Eight-week-old male ApoE ${ }^{-/-}$mice on a C57BL/6 background (Jackson Laboratory, Bar Harbor, ME, USA) were fed a standard chow diet, pretreated with anakinra for 4 weeks, then fed an atherogenic diet containing $35 \mathrm{kcal} \%$ fat, 1.25\% cholesterol, and 0.5\% sodium cholic acid (D12236; Research Diets Inc., 
New Brunswick, NJ, USA) for 12 weeks with continued anakinra treatment. During the intervention period, the $\mathrm{ApoE}^{-/-}$mice were divided into the following four treatment groups, where each drug was

administered daily by intraperitoneal injection to assess the potential dose-dependent anti-atherosclerosis effects of anakinra: (1) control ( $n=10$, normal saline, $154 \mathrm{mmol} / \mathrm{L} \mathrm{NaCl}),(2)$ anakinra $10 \mathrm{mg} / \mathrm{kg}(\mathrm{n}=10)$, (3) anakinra $25 \mathrm{mg} / \mathrm{kg}(\mathrm{n}=10)$, and (4) anakinra $50 \mathrm{mg} / \mathrm{kg}(\mathrm{n}=10)$. The number of mice per group was selected with a previous atherogenic experimental plan study [19]. Random sequence was created using Excel 2013 (Microsoft, Redmond, WA, USA). Mice were maintained in a controlled climate room with a light-dark cycle (12:12), and body weight and food intake were monitored once a week. At the time of euthanasia, mice were anesthetized by zoletil $(30 \mathrm{mg} / \mathrm{kg}$, i.p.) with xylazine (10 mg/kg, i.p.), and blood was collected by cardiac puncture after overnight fasting. Serum samples were used for triglyceride and cholesterol analyses. Aorta, liver, visceral fat, and muscle tissues were harvested for further histopathological analysis.

The aortic root was dissected longitudinally for the en face method and stained with oil-red 0 to measure the aortic atherosclerotic lesions. Section images were analyzed using an Olympus BX51 imaging system (Olympus, Tokyo, Japan) and quantified with Image-Pro Plus 6.0 software (MediaCybernetics, Bethesda, MD, USA). The area of atherosclerotic plaque was expressed as a percentage of the entire area of the aorta. The protocol for staining plaque fibrosis was described previously [20].

\section{Immunofluorescent staining for CD68}

The number of M1 macrophages in the aortic fat tissue was evaluated as the number of CD68 + cells per $1 \mathrm{~mm}^{2}$, using an anti-mouse CD68 antibody (1:200 dilution, Abcam, Cambridge, MA, USA).

\section{THP-1-conditioned media and induction of NLRP3 inflammasome expression in human umbilical vein endothelial cells (HUVECs), rat aortic smooth muscle cells (RAOSMCs), and 3T3-L1 cells}

The human monocytic cell line, THP-1 $\left(5.5 \cdot 10^{6}\right.$ cells/well) was maintained in RPMI 1640 (Gibco, Thermo Fisher Scientific, USA) supplemented with $10 \%$ fetal bovine serum (Gibco, Thermo Fisher Scientific, USA), at $37{ }^{\circ} \mathrm{C}$ and $5 \% \mathrm{CO}_{2}$. To induce THP-1 cells to differentiate to macrophages, they were treated overnight with 100 nM phorbol-12-myristate-13-acetate (PMA; Sigma, Catalog \#: P1585, Buchs, Switzerland), which was then replaced with fresh growth media and cultures were incubated for a further $24 \mathrm{~h}$.

To examine the effect of anakinra on NLRP3 inflammasome activity in atherosclerosis, HUVECs (Lonza, San Diego, CA, USA), RAOSMCs (Bio-bud, Seoul, Republic of Korea), and 3T3-L1 cells (ATCC, Manassas, VA, USA) were used. Differentiated PMA-treated THP-1 cells were incubated for $6 \mathrm{~h}$ with $1 \mu \mathrm{g} / \mathrm{mL}$ lipopolysaccharide (LPS; Sigma, Catalog \#: L2880, Buchs, Switzerland) and $100 \mathrm{ng} / \mathrm{mL}$ TNF-a (ProSpec, Catalog \#: Cyt-223-b, Ness-Ziona, Israel). Cells were removed (3000 rpm, $5 \mathrm{~min}, 0.22 \mu \mathrm{m}$ filter) and supernatants (conditioned medium) were harvested. Then, the conditioned medium was added to preplated HUVECs, RAOSMCs, and 3T3-L1 in the presence or absence of anakinra.

Reverse transcription-quantitative polymerase chain reaction (RT-qPCR) 
The relative levels of mRNA transcripts for NLRP3, IL-1 $\beta$, IL-6, monocyte chemoattractant protein-1 (MCP1), ICAM-1, and matrix metalloproteinase-9 (MMP-9) were assessed by RT-qPCR using the $\beta$-actin gene as reference gene. The sequences of the primers used are shown in online appendices [Supplementary Information Table 1].

\section{Western blot analysis}

Proteins were extracted from cells, and lysates containing appropriate amounts of protein were resolved on $10 \%$ SDS-polyacrylamide gels and transferred to polyvinylidene difluoride membranes. Nonspecific binding was blocked in $5 \%$ bovine serum albumin for $2 \mathrm{~h}$ at room temperature. Membranes were incubated overnight at $4{ }^{\circ} \mathrm{C}$ with primary antibodies (online appendices: Supplementary Information Table 2). Next, the membranes were washed and then incubated for $1 \mathrm{~h}$ at room temperature with horseradish peroxide-conjugated anti-rabbit or anti-mouse secondary antibodies (Santa Cruz Biotechnology, CA, USA).

\section{RAOSMC migration assays}

RAOSMC migration capacity was assessed by two-dimensional wound healing assays. For the wound healing assay, cells were seeded at a density of $2 \cdot 10^{5}$ cells/well in 12-well plates and starved with serum-free DMEM media for $24 \mathrm{~h}$ before experiments. Linear wounds were made by scratching with a $1000 \mathrm{~mL}$ pipette tip. RAOSMCs were allowed to migrate for $24 \mathrm{~h}$ in the presence or absence of plateletderived growth factor (PDGF; $10 \mathrm{ng} / \mathrm{mL})$ and anakinra $(1000 \mathrm{ng} / \mathrm{mL})$ at $37^{\circ} \mathrm{C}$; then images of the migrated RAOSMCs were acquired using an inverted microscope (Olympus).

\section{Statistical analysis}

All data are expressed as mean \pm standard error of the mean. Statistical significance was determined using the analysis of variance (ANOVA) with Tukey's post hoc analysis for multiple group comparison. Values of two-sided $P<0.05$ were considered significant. Statistical analyses were performed using SPSS Statistics for Windows (version 24.0; IBM Corp., Armonk, NY, USA). Illustration was created using the online software tool (BioRender, Toronto, Ontario, Canada).

\section{Results}

\section{Focus on atherosclerosis}

\section{Anakinra reduces the atherosclerotic plaque area in $\mathrm{ApoE}^{-/-}$mice}

Images of the aortic arches with atheromatous plaque from representative individuals of each group are shown in Fig. 1. Atheromatous plaque accumulation in the aortic arch of atherogenic dieted ApoE ${ }^{-/-}$mice $^{-}$ was reduced by $30.6 \%$ and $25.2 \%$ at the 25 and $50 \mathrm{mg} / \mathrm{kg}$ doses of anakinra, respectively (both $P<0.05$, compared with vehicle-treated $\mathrm{ApoE}^{-/-}$mice). Serum triglycerides (TG) were significantly decreased in mice treated with $50 \mathrm{mg} / \mathrm{kg}$ anakinra $(P<0.05)$, whereas there was no change in serum total cholesterol. 
The dose-dependent effect of anakinra on the activation of the NLRP3 inflammasome and upregulated expression of inflammatory adhesion molecules in HUVECs

Expression of mRNAs for NLRP3, IL-1ß, IL-6, ICAM-1, and MCP-1 were significantly increased in HUVECs stimulated with conditioned medium from differentiated LPS- and TNF-a-stimulated THP-1 macrophages compared with unmanipulated control HUVECs (all $P<0.001$ ) (Fig. 2). Expression of each mRNA in stimulated HUVECs after each dose of anakinra was compared with the positive control (without anakinra treatment).

The expression of NLRP3 mRNA was not significantly changed by treatment with anakinra. The expression of IL-1 $\beta$ mRNA was markedly decreased by anakinra doses of $100 \mathrm{ng} / \mathrm{mL}, 500 \mathrm{ng} / \mathrm{mL}$, and $1000 \mathrm{ng} / \mathrm{mL}$, respectively (all $P<0.01$ ). IL-6 mRNA expression was also significantly decreased at high concentrations of anakinra ( $500 \mathrm{ng} / \mathrm{mL}$ and $1000 \mathrm{ng} / \mathrm{mL}$, both $P<0.05)$. However, expression of ICAM-1 and MCP-1 mRNAs in HUVECs was not significantly changed by anakinra.

\section{The dose-dependent effect of anakinra on the activated NLRP3 inflammasome and upregulated expression of angiogenesis molecules in RAOSMCs}

Stimulation of RAOSMCs with the supernatants from LPS- and TNF-a-stimulated differentiated THP-1 macrophages induced significantly increased expression of mRNAs for NLRP3, IL-1 $\beta$, IL-6, and MMP-9. RAOSMCs treated with relatively high doses of anakinra showed a significant decrease in expression of NLRP3 mRNA (1000 ng/mL), IL-1 $\beta$ (500 ng/mL and $1000 \mathrm{ng} / \mathrm{mL})$, and IL-6 $(1000 \mathrm{ng} / \mathrm{mL})$ compared with no anakinra treatment (all $P<0.05$ ) (Fig. 3). The expression of MMP-9 was dose-dependently reduced by $100 \mathrm{ng} / \mathrm{mL}, 500 \mathrm{ng} / \mathrm{mL}$, and $1000 \mathrm{ng} / \mathrm{mL}$ doses of anakinra (all $P<0.05$, compared with the no anakinra treatment group).

\section{The effect of anakinra on the p38 mitogen-activated protein kinase (MAPK)/nuclear factor-kB (NF-KB) pathway in RAOSMCs and HUVECs}

The results of the western blot analysis demonstrated that treatment of RAOSMCs for $5,10,15,30$, or 60 min with the supernatant from LPS- and TNF-a-stimulated THP-1 macrophages induced increased expression of phospho-NF-KB p65 protein, which reached peak levels after $15 \mathrm{~min}$ and remained elevated until $60 \mathrm{~min}$. Exposure of RAOSMCs to the supernatant from the LPS- and TNF-a-stimulated differentiated THP-1 macrophages for $60 \mathrm{~min}$ also induced significantly increased expression of phospho-p38 MAPK. Because NF-KB plays an important role in regulation of the vascular inflammatory responses, we examined the effect of anakinra on the phosphorylation of NF-KB p65, an essential step in the activation of NF-KB. Activation of the $\mathrm{p} 38 \mathrm{MAPK} / \mathrm{NF}-\mathrm{KB}$ pathway in RAOSMCs was inhibited by treatment with anakinra $(1000 \mathrm{ng} / \mathrm{mL})(P<0.05)$ (Fig. 4). In HUVECs, the phosphorylation of p65 and extracellular-regulated kinases (ERK) $1 / 2$ showed a tendency to decrease in the anakinra-treated group compared with the control group, but this was not significant (data not shown).

\section{Anakinra inhibits migration of RAOSMCs}


To understand better the effects of anakinra on vascular injury and repair, a migration/wound healing assay was performed using RAOSMCs stimulated by PDGF. A representative field is shown in Fig. $\mathbf{5}$. Migration capability measured by the wound-healing assay revealed that anakinra caused a significant $57 \%$ reduction in RAOSMC migration.

\section{Focus on fat}

\section{Immunofluorescent staining of CD68 in visceral adipose tissue}

Immunofluorescent staining for CD68, which is a surface marker of M1 macrophages, indicated that the number of M1 macrophages in visceral adipose tissue of mice tended to decrease in mice treated with 25 $\mathrm{mg} / \mathrm{kg}$ and $50 \mathrm{mg} / \mathrm{kg}$ anakinra compared with controls, but these differences were not significant (Fig. 6).

\section{The dose-dependent effect of anakinra on the activated NLRP3 inflammasome and upregulated expression of inflammatory molecules in 3T3-L1 adipocytes}

The results of these experiments are shown in Fig. 7. Compared with the negative control group, 3T3-L1 adipocytes stimulated with supernatant from LPS- and TNF-a-stimulated THP-1 macrophages showed significant upregulation of mRNA for NLRP3, IL-1 $3, \mathrm{IL}-6$, and MCP-1 (all $P<0.001$ ). Treatment of 3T3-L1 adipocytes with $100 \mathrm{ng} / \mathrm{mL}, 500 \mathrm{ng} / \mathrm{mL}$, and $1000 \mathrm{ng} / \mathrm{mL}$ anakinra significantly reduced the levels of NLRP3 mRNA (all $P<0.05$ ). The levels of MCP-1 mRNA were also significantly decreased at all doses of anakinra (all $P<0.05$ ). In contrast, although the levels of IL-1 $\beta$ and IL-6 mRNAs showed a tendency to decrease at high doses of anakinra, this decrease was not significant.

\section{Expression of phosphorylated c-Jun N-terminal kinase ( $\mathrm{p}-\mathrm{JNK}$ ), $\mathrm{p}-\mathrm{p} 38$, and $\mathrm{p}$-ERK in the liver and visceral fat tissue}

The expression of p-ERK in liver tissue tended to decrease at high doses of anakinra $(25 \mathrm{mg} / \mathrm{kg}$ and 50 $\mathrm{mg} / \mathrm{kg} /$ day) compared with the control group, but this was not significant. Similarly, the expression of $\mathrm{p}$ ERK in visceral adipose tissue showed a nonsignificant tendency to decrease in the high-dose anakinra (50 mg/kg/day) group compared with the control group. In terms of the expression of p-JNK in fat tissue, the anakinra $25 \mathrm{mg} / \mathrm{kg} /$ day group showed an increase compared with the control group but this was not dose-dependent (data not shown).

\section{Discussion}

This study demonstrated that IL-1 blockade with anakinra significantly reduced atherosclerotic plaque formation and progression in the aortic arch of $\mathrm{ApoE}^{-/-}$mice fed an atherogenic diet. In addition, anakinra suppressed the expression of inflammatory biomarkers, such as IL-6, MMP-9, and MCP-1 in HUVEC, RAOSMC, and 3T3-L1 adipocytes, suggesting that anakinra treatment could be a useful strategy for blocking the inflammatory signals mediating the process of atherosclerosis and systematic 
inflammation. Anakinra produced a significant dose-dependent decrease in MMP-9 mRNA expression, and also decreased migration of RAOSMCs, which is a model of vascular remodeling.

Statins are the cornerstone of lipid management for reducing cardiovascular risk. A reduction in LDL-C of $1 \mathrm{mmol} / \mathrm{L}(38.9 \mathrm{mg} / \mathrm{dL})$ can be expected to reduce the relative risk of major cardiovascular events and all-cause mortality by $22 \%$ and $10 \%$, respectively [21]. However, $40-60 \%$ of patients taking statins are still at risk of fatal cardiovascular events despite achieving their target LDL-C levels [22]. This implies that further treatment strategies are needed following standard lipid-lowering treatment to reduce this residual risk. Cumulative evidence links chronic inflammation with atherosclerotic CVD [23, 24]. The prototypical proinflammatory cytokine, IL-1, specifically contributes to the formation, growth, and rupture of atherosclerotic plaques in ischemic CVD $[25,26]$. Caspase-1 plays an important role in the activation of IL-1 $\beta$. Activation of the NLRP3 inflammasome leads to conversion of pro-caspase- 1 to the active enzyme, caspase- 1 . Active caspase- 1 then cleaves pro-IL-1 $\beta$ and releases the biologically active "mature" IL-1 [ 27 , 28]. Generation of mature IL-1 $\beta$ from bystander cells or extracellular pro-IL-1 $\beta$ contributes to an autoactivation loop of inflammatory responses, i.e., activation of immune cells, and release of proinflammatory cytokines and danger-associated molecular patterns (e.g., adenosine triphosphate, palmitate, ceramide, and cholesterol), eventually leading to cell death [29].

In light of these associations between IL- $1 \beta$ and CVD, the recently published findings of the Canakinumab Anti-inflammatory Thrombosis Outcome Study (CANTOS) suggested that anti-inflammatory treatment in parallel with statin treatment could be a strategy for the secondary prevention of recurrent major cardiovascular events in patients with previous MI [30]. That study investigated the effect of adding canakinumab, a monoclonal antibody targeting IL-1 $\beta$, to statin treatment, on the incidence of classical 3point major adverse cardiovascular events (MACE) in more than 10,000 patients with previous MI. The findings showed that $150 \mathrm{mg}$ of canakinumab reduced the risk of 3-point MACE by $15 \%$ compared with placebo in patients with well-controlled LDL-C levels. However, the effect of reducing the absolute risk was not large: 156 patients had to be treated with canakinumab for 1 year to avoid the primary endpoint, MACE. Moreover, the main effect was driven by the reduction in the frequency of MI among the 3-point MACE, and cardiovascular mortality and cerebrovascular events did not differ between groups. Although several preliminary preclinical and clinical trials show that IL-1 blockers have potential benefits in atherosclerosis, cerebral infarction, acute $\mathrm{Ml}$, and heart failure, the data are inadequate to draw consistent conclusions [31-33]. Therefore, additional investigations are needed to determine the most effective tool for inhibiting IL-1 or IL-1 $\beta$ in anti-atherosclerosis treatment.

Anakinra was the first drug targeting IL-1 to be approved as a therapeutic agent for rheumatoid arthritis, and has now been recognized to have excellent long-term safety for treatment of chronic diseases such as cryopyrin-associated periodic syndromes, which are related to pathogenic variants in IL-1 [34, 35]. Moreover, anakinra inhibited expression of ICAM-1 and E-selectin in monocytes and improved endothelial dysfunction through diminishing endoplasmic reticulum stress and reducing infiltration of inflammatory cells. It has also been reported to reduce ischemia-induced neovascularization in diabetic rodents [3638]. 
The $\mathrm{ApoE}^{-/-}$mouse is a well-established animal model that has been applied extensively to research on the mechanism of atherosclerosis development [39]. We used this animal model to explore the utility and mechanisms of an anti-IL-1 $\beta$ treatment in atherosclerosis. In $\mathrm{ApoE}^{-/-}$mice, even though statins produce $30-60 \%$ attenuation of atherosclerosis progression $[40-42]$. $\mathrm{ApoE}^{-/-}$mice have increased very-low density lipoprotein and increased LDL-C, an animal model that may reflect the poorly controlled residual risk of CVD in humans even during statin treatment. Interestingly, the present study showed that anakinra significantly reduced the level of serum triglycerides in $\mathrm{ApoE}^{-/-}$mice.

The present study also showed that anakinra treatment reduced the burden of lipid-laden atherosclerotic plaques in $\mathrm{ApoE}^{-/-}$mice by $30 \%$ compared with the control group. Similar results have been obtained using gevokizumab, a monoclonal antibody against IL- $1 \beta$, which reduced plaque area in the aortic arch of $\mathrm{ApoE}^{-/-}$mice by about 30\% [43]. Moreover, anakinra induced a dose-dependent decrease in the number of $\mathrm{CD} 68+$ cells, widely used as a marker of the macrophage lineage, in fat tissue. Because inflamed fat tissue produces "bad" cytokines that promote systemic chronic inflammation, anakinra could provide a means to reduce both atherosclerotic plaques and inflammation in fat tissue [44-47].

The present study found that the expression of IL-6 mRNA was lower in anakinra-treated HUVECs and RAOSMCs compared with control untreated cells. In 3T3-L1 adipocytes, anakinra treatment resulted in significantly lower MCP-1 mRNA expression compared with the control group. In RAOSMCs, anakinra induced a significant dose-dependent decrease in MMP-9 expression, and also decreased RAOSMC migration, which reduces wound healing and the progress of vascular restenosis. A previous study using mice and rats with induced acute $\mathrm{MI}$ reported a 13\% reduction in the infarct size in animals treated with $100 \mathrm{mg} / \mathrm{kg}$ anakinra, which suggested that IL-1 could play an important role in post-injury cardiac remodeling $[13,38]$. Taken together, these results suggest that anakinra inhibits the functions of IL- 1 , thereby reducing proinflammatory cytokine release and inflammatory cell recruitment, decreasing plaque size, and stabilizing the lipid-laden plaques, and anakinra therefore inhibits almost all stages of atherosclerosis.

This study has several limitations. First, the protocol specified that anakinra was administered at all stages, starting during feeding of a normal chow diet for the initial 4 weeks and continuing during feeding of an atherogenic diet for the last 12 weeks. This differs from other in vivo studies that used statin treatment only during feeding of an atherogenic diet. However, a previous study treating $\mathrm{ApoE}^{-/-}$mice with IL-1 receptor antagonist showed that the drug affected the earlier stages of plaque formation [48]. Second, we wanted to clarify the effect of anakinra independent of the well-known effects of statin treatment in $\mathrm{ApoE}^{-/-}$mice; thus, the combined effect of anakinra and statin could not be assessed. It is expected that anti-inflammatory mechanism-based treatment, anakinra, could additional effect on the inhibition of the initiation and progression of atherosclerosis in a variety of ways that are not possible with only some established lipid lowering agents. Therefore, it would be helpful to conduct further studies in this animal model to investigate the anti-atherosclerosis effects of different doses and durations of combination treatment with anakinra and standard lipid-lowering agents. Third, anakinra showed a 
significant decrease in IL-1 $\beta$ mRNA expression in HUVEC, but did not show the clear effect on the expressions of ICAM-1 or MCP-1. Whether these were some characteristics of endothelial cells per se or the result of methodological issues such as anakinra dosage or time for treatment has not been confirmed in this study, but it could be possible that other factors related to the subsequent process of endothelial cells were intervened. Based on several previous studies showing increased gene expressions of cell adhesion molecules or monocyte-attracting chemokines, a complex manner such as, the way of stimulus, growth factors, and collagens were involved in modulation of endothelial cells $[37,49]$.

\section{Conclusions}

In conclusion, we demonstrated that the IL-1 blocker anakinra produced a $30 \%$ reduction in atherosclerotic plaque area in $\mathrm{ApoE}^{-/-}$mice, and confirmed its anti-inflammatory effects in experiments using RAOSMC, HUVEC, and 3T3-L1 adipocytes. Atherosclerotic CVDs have a complicated pathogenesis, so identifying the best way to ameliorate the residual risk after the standard therapy for preventing CVD events remains a challenge. Our results are mediated by blocking chronic inflammation in atherosclerosis via the IL-1 cascade, and could support the extended use of anakinra and other novel targeted cytokinebased therapies together with lipid-lowering agents to overcome the residual risk of major cardiovascular events after standard treatment.

\section{Declarations}

\section{Funding}

This work was supported by the Medical Research Center (MRC) through the National Research Foundation of Korea (NRF) funded by the Ministry of Science [grant number NRF-2018R1A5A2024425] to Sung Hee Choi.

\section{Conflicts of interest}

The authors declare that they have no known competing financial interests or personal relationships that could influence this work. The funding source did not have any input in the study design, data collection, interpretation of the results, preparation of the manuscript, or publishing decision.

\section{Author's Contributions}

EJK: Conceptualization, methodology, validation, formal analysis and writing - original draft. BRK: Experiment, investigation and writing - original draft. J-IL, Y-KL, TJO and HCJ Investigation and writing review. SHC: Conceptualization, methodology and writing - original draft, resources, supervision, project administration and funding acquisition.

\section{Acknowledgments}

Only the authors listed on the manuscript contributed to this research. 
Availability of data and material The datasets used and/or analyzed during the current study are available from the corresponding author on reasonable request.

Supplementary Information The online supplementary information is available at http://doi.org/10.6084/m9.figshare.14510478

Ethics Approval All animal experiment protocols were approved by the Seoul National University Bundang Hospital Institutional Animal Care and Use Committee (BA1511-188/070 - 01).

Consent to Participate Not applicable. We did not include any human participants.

Consent for Publication All authors have approved the final submitted version and publication.

\section{References}

1. Benjamin EJ, Blaha MJ, Chiuve SE, et al. Heart Disease and Stroke Statistics-2017 Update: A Report From the American Heart Association. Circulation. 2017;135:e146-603.

2. Biondi-Zoccai GG, Abbate A, Liuzzo G, Biasucci LM. Atherothrombosis, inflammation, and diabetes. J Am Coll Cardiol. 2003;41:1071-7.

3. Maffia P, Grassia G, Di Meglio P, et al. Neutralization of interleukin-18 inhibits neointimal formation in a rat model of vascular injury. Circulation. 2006;114:430-7.

4. Wu G, Mannam AP, Wu J, et al. Hypoxia induces myocyte-dependent COX-2 regulation in endothelial cells: role of VEGF. Am J Physiol Heart Circ Physiol. 2003;285:H2420-9.

5. Isoda K, Shiigai M, Ishigami N, et al. Deficiency of interleukin-1 receptor antagonist promotes neointimal formation after injury. Circulation. 2003;108:516-8.

6. Wang X, Feuerstein GZ, Gu IL, Lysko PG, Yue TL. Interleukin-1 beta induces expression of adhesion molecules in human vascular smooth muscle cells and enhances adhesion of leukocytes to smooth muscle cells. Atherosclerosis. 1995;115:89-98.

7. Broz P, Dixit VM. Inflammasomes: mechanism of assembly, regulation and signaling. Nat Rev Immunol. 2016;16:407-20.

8. Abbate A, Toldo S, Marchetti C, Kron J, Van Tassell BW, Dinarello CA. Interleukin-1 and the Inflammasome as Therapeutic Targets in Cardiovascular Disease. Circ Res. 2020;126:1260-80.

9. Ridker PM, Libby P, MacFadyen JG, et al. Modulation of the interleukin-6 signalling pathway and incidence rates of atherosclerotic events and all-cause mortality: analyses from the Canakinumab Anti-Inflammatory Thrombosis Outcomes Study (CANTOS). Eur Heart J. 2018;39:3499-507.

10. Van Tassell BW, Raleigh JM, Abbate A. Targeting interleukin-1 in heart failure and inflammatory heart disease. Curr Heart Fail Rep. 2015;12:33-41.

11. Van Tassell BW, Toldo S, Mezzaroma E, Abbate A. Targeting interleukin-1 in heart disease. Circulation. 2013;128:1910-23. 
12. Emmi G, Urban ML, Imazio M, et al. Use of Interleukin-1 Blockers in Pericardial and Cardiovascular Diseases. Curr Cardiol Rep. 2018;20:61.

13. Szekely Y, Arbel Y. A Review of Interleukin-1 in Heart Disease: Where Do We Stand Today? Cardiol Ther. 2018;7:25-44.

14. Van Tassell BW, Buckley LF, Carbone S, et al. Interleukin-1 blockade in heart failure with preserved ejection fraction: rationale and design of the Diastolic Heart Failure Anakinra Response Trial 2 (DHART2). Clin Cardiol. 2017;40:626-32.

15. Garces K. Anakinra: interleukin-1 receptor antagonist therapy for rheumatoid arthritis. Issues Emerg Health Technol. 2001;1:1-4.

16. Van Tassell BW, Abouzaki NA, Oddi Erdle C, et al. Interleukin-1 Blockade in Acute Decompensated Heart Failure: A Randomized, Double-Blinded, Placebo-Controlled Pilot Study. J Cardiovasc Pharmacol. 2016;67:544-51.

17. Van Tassell BW, Arena R, Biondi-Zoccai G, et al. Effects of interleukin-1 blockade with anakinra on aerobic exercise capacity in patients with heart failure and preserved ejection fraction (from the DHART pilot study). Am J Cardiol. 2014;113:321-7.

18. Ikonomidis I, Lekakis JP, Nikolaou M, et al. Inhibition of interleukin-1 by anakinra improves vascular and left ventricular function in patients with rheumatoid arthritis. Circulation. 2008;117:2662-9.

19. Dhar-Mascareno M, Rozenberg I, Iqbal J, Hussain MM, Beckles D, Mascareno E. Hexim1 heterozygosity stabilizes atherosclerotic plaque and decreased steatosis in ApoE null mice fed atherogenic diet. Int J Biochem Cell Biol. 2017;83:56-64.

20. Tian $Y$, Chen T, Wu Y, et al. Pioglitazone stabilizes atherosclerotic plaque by regulating the Th17/Treg balance in AMPK-dependent mechanisms. Cardiovasc Diabetol. 2017;16:140.

21. Cholesterol Treatment Trialists' (CTT). Collaboration, Fulcher J, O'Connell R. M. et al. Efficacy and safety of more intensive lowering of LDL cholesterol: a meta-analysis of data from 170,000 participants in 26 randomised trials. Lancet. 2015;385:1397-405.

22. Reith C, Armitage J. Management of residual risk after statin therapy. Atherosclerosis. 2016;245:161-70.

23. Ridker PM. C-reactive protein and the prediction of cardiovascular events among those at intermediate risk: moving an inflammatory hypothesis toward consensus. J Am Coll Cardiol. 2007:49:2129-38.

24. Libby P, Ridker PM, Maseri A. Inflammation and atherosclerosis. Circulation. 2002;105:1135-43.

25. Buckley LF, Abbate A. Interleukin-1 blockade in cardiovascular diseases: a clinical update. Eur Heart J. 2018;39:2063-9.

26. Peiró C, Lorenzo Ó, Carraro R, Sánchez-Ferrer CF. IL-1beta Inhibition in Cardiovascular Complications Associated to Diabetes Mellitus. Front Pharmacol. 2017;8:363.

27. Cremer PC, Kumar A, Kontzias A, et al. Complicated Pericarditis: Understanding Risk Factors and Pathophysiology to Inform Imaging and Treatment. J Am Coll Cardiol. 2016;68:2311-28. 
28. Dinarello CA. Interleukin-1 in the pathogenesis and treatment of inflammatory diseases. Blood. 2011;117:3720-32.

29. Baroja-Mazo A, Martín-Sánchez F, Gomez Al, et al. The NLRP3 inflammasome is released as a particulate danger signal that amplifies the inflammatory response. Nat Immunol. 2014;15:738-48.

30. Ridker PM, Everett BM, Thuren T, et al. Antiinflammatory Therapy with Canakinumab for Atherosclerotic Disease. N Engl J Med. 2017;377:1119-31.

31. Crossman D, Rothman AMK. Interleukin-1 beta inhibition with canakinumab and reducing lung cancer-subset analysis of the canakinumab anti-inflammatory thrombosis outcome study trial (CANTOS). J Thorac Dis. 2018;10:3084-7.

32. Harouki N, Nicol L, Remy-Jouet I, et al. The IL-1beta Antibody Gevokizumab Limits Cardiac Remodeling and Coronary Dysfunction in Rats With Heart Failure. JACC Basic TransI Sci. 2017;2:418-30.

33. Xia YY, Song SW, Min Y, et al. The effects of anakinra on focal cerebral ischemic injury in rats. CNS Neurosci Ther. 2014;20:879-81.

34. Kullenberg T, Löfqvist M, Leinonen M, Goldbach-Mansky R, Olivecrona H. Long-term safety profile of anakinra in patients with severe cryopyrin-associated periodic syndromes. Rheumatology. 2016;55:1499-506.

35. Dinarello CA, Simon A, Van der Meer JW. Treating inflammation by blocking interleukin-1 in a broad spectrum of diseases. Nat Rev Drug Discov. 2012;11:633-52.

36. Vallejo S, Palacios E, Romacho T, Villalobos L, Peiró C, Sánchez-Ferrer CF. The interleukin-1 receptor antagonist anakinra improves endothelial dysfunction in streptozotocin-induced diabetic rats. Cardiovasc Diabetol. 2014;13:158.

37. Shikama Y, Aki N, Hata A, Nishimura M, Oyadomari S, Funaki M. Palmitate-stimulated monocytes induce adhesion molecule expression in endothelial cells via IL-1 signaling pathway. J Cell Physiol. 2015;230:732-42.

38. Abbate A, Salloum FN, Vecile E, et al. Anakinra, a recombinant human interleukin-1 receptor antagonist, inhibits apoptosis in experimental acute myocardial infarction. Circulation. 2008;117:2670-83.

39. Plump AS, Smith JD, Hayek T, et al. Severe hypercholesterolemia and atherosclerosis in apolipoprotein E-deficient mice created by homologous recombination in ES cells. Cell. 1992;71:34353.

40. Bot I, Jukema JW, Lankhuizen IM, van Berkel TJ, Biessen EA. Atorvastatin inhibits plaque development and adventitial neovascularization in ApoE deficient mice independent of plasma cholesterol levels. Atherosclerosis. 2011;214:295-300.

41. Monetti $\mathrm{M}$, Canavesi $\mathrm{M}$, Camera $\mathrm{M}$, et al. Rosuvastatin displays anti-atherothrombotic and antiinflammatory properties in apoE-deficient mice. Pharmacol Res. 2007;55:41-9.

42. Chen J, Li D, Schaefer R, Mehta JL. Cross-talk between dyslipidemia and renin-angiotensin system and the role of LOX-1 and MAPK in atherogenesis studies with the combined use of rosuvastatin and 
candesartan. Atherosclerosis. 2006;184:295-301.

43. Bhaskar V, Yin J, Mirza AM, et al. Monoclonal antibodies targeting IL-1 beta reduce biomarkers of atherosclerosis in vitro and inhibit atherosclerotic plaque formation in Apolipoprotein E-deficient mice. Atherosclerosis. 2011;216:313-20.

44. Ghigliotti G, Barisione C, Garibaldi S, et al. Adipose tissue immune response: novel triggers and consequences for chronic inflammatory conditions. Inflammation. 2014;37:1337-53.

45. Pietiläinen KH, Róg T, Seppänen-Laakso T, et al. Association of lipidome remodeling in the adipocyte membrane with acquired obesity in humans. PLoS Biol. 2011;9:e1000623.

46. Kolodgie FD, Virmani R, Burke AP, et al. Pathologic assessment of the vulnerable human coronary plaque. Heart. 2004;90:1385-91.

47. Virmani R, Kolodgie FD, Burke AP, Farb A, Schwartz SM. Lessons from sudden coronary death: a comprehensive morphological classification scheme for atherosclerotic lesions. Arterioscler Thromb Vasc Biol. 2000;20:1262-75.

48. Isoda K, Sawada S, Ishigami N, et al. Lack of interleukin-1 receptor antagonist modulates plaque composition in apolipoprotein E-deficient mice. Arterioscler Thromb Vasc Biol. 2004;24:1068-73.

49. Eissner G, Lindner H, Reisbach G, Klauke I, Holler E. Differential modulation of IL-1-induced endothelial adhesion molecules and transendothelial migration of granulocytes by G-CSF. Br J Haematol. 1997;97:726-33.

\section{Figures}

a

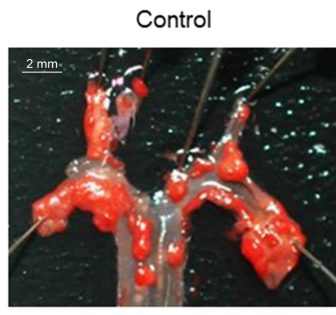

$10 \mathrm{mg} / \mathrm{kg} / \mathrm{day}$

$25 \mathrm{mg} / \mathrm{kg} / \mathrm{day}$

$50 \mathrm{mg} / \mathrm{kg} / \mathrm{day}$
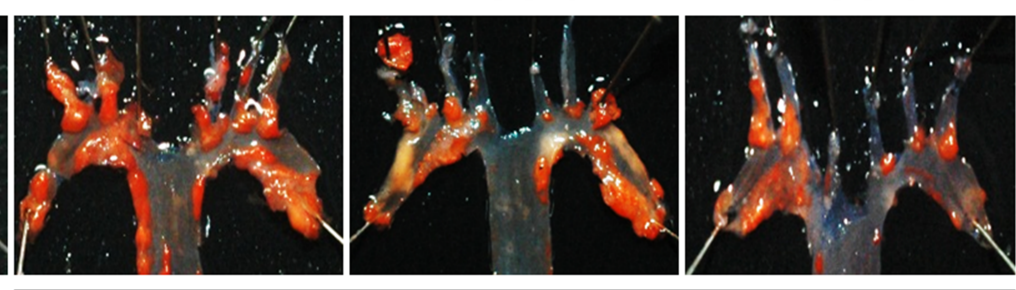

Anakinra

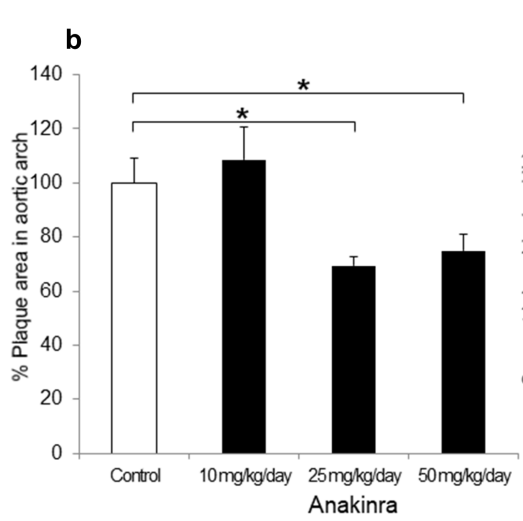

c

d
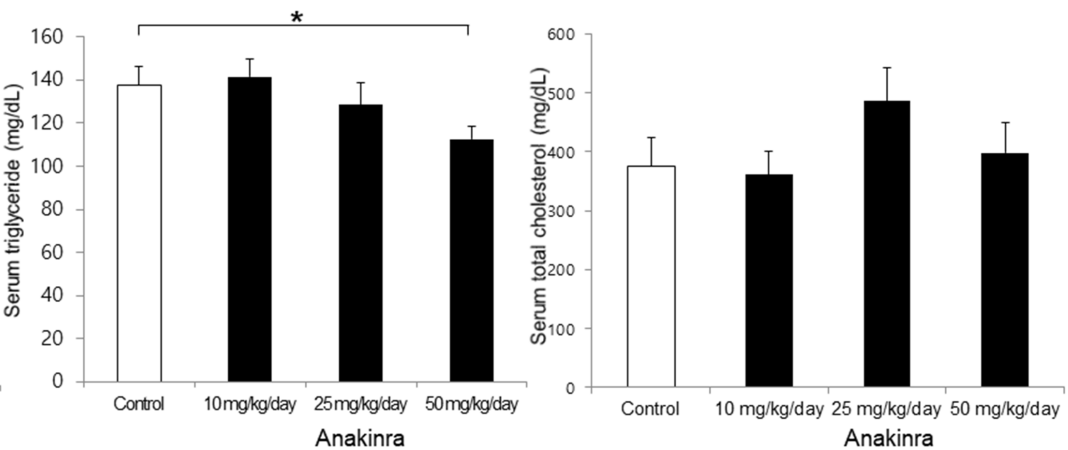
Figure 1

The effect of anakinra on atherosclerosis. a Area of atheromatous plaque in the aortic arch in ApoE-/mice was measured using the en face method and expressed as a percentage of oil-red 0-positive pixels compared with the control group. b Plaque area was significantly reduced by $30.6 \%$ and $25.2 \%$ compared with the control group by treatment with $25 \mathrm{mg} / \mathrm{kg}$ and $50 \mathrm{mg} / \mathrm{kg}$ anakinra, respectively. c In ApoE-/mice, serum triglyceride level was decreased by treatment with $50 \mathrm{mg} / \mathrm{kg}$ anakinra. $\mathrm{d}$ In ApoE-/- mice, serum total cholesterol did not differ significantly between groups. ${ }^{*} P<0.05,+P<0.01, \pm P<0.001$ by the analysis of variance (ANOVA) with Tukey's post hoc analysis for multiple group comparison.
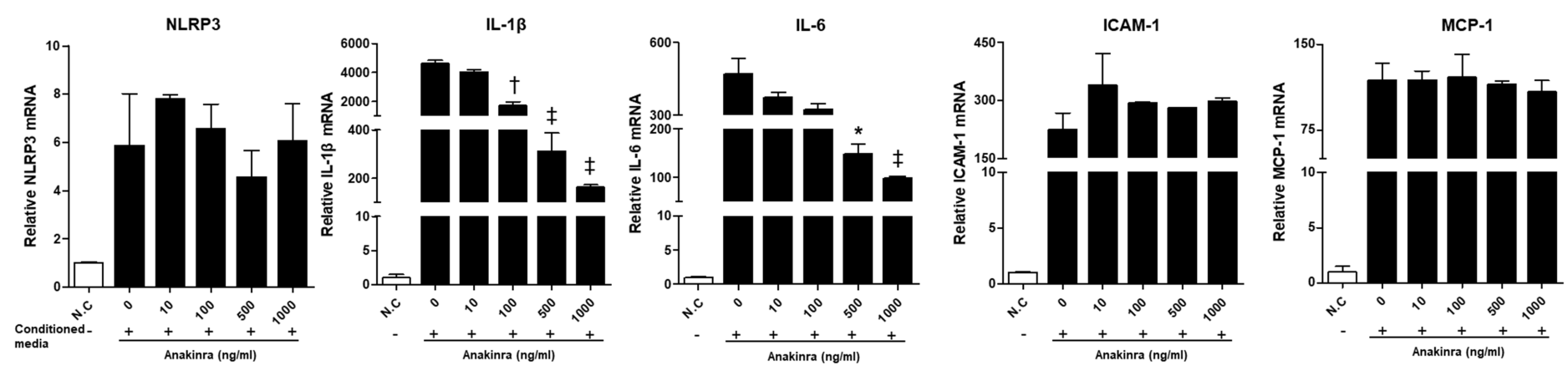

\section{Figure 2}

The effect of dose-dependent anakinra on the activated NLRP3 inflammasome and upregulated expression of inflammatory molecules in HUVECs after stimulation with conditioned media from differentiated LPS and TNFa stimulated THP-1 macrophages. Gene expressions were analyzed by qRT$P C R$. ${ }^{*}<0.05,+P<0.01, \neq P<0.001$ compared with control group (anakinra $0 \mathrm{ng} / \mathrm{mL}$ ) using the analysis of variance (ANOVA) with Tukey's post hoc analysis for multiple group comparison. 

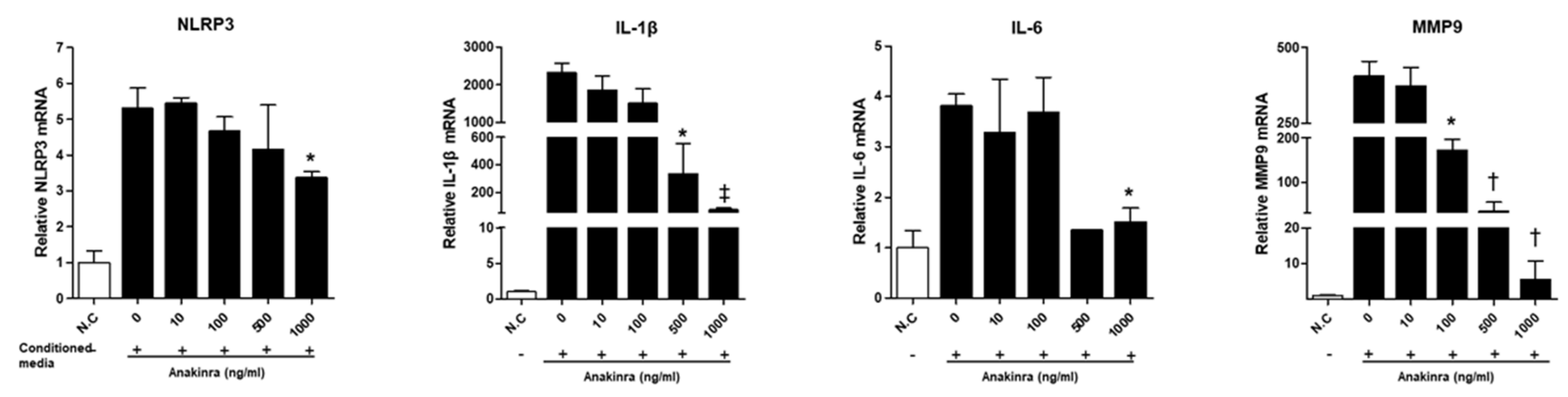

\section{Figure 3}

The effect of dose-dependent anakinra on the activated NLRP3 inflammasome and upregulated expression of inflammatory molecules in RAOSMCs after stimulation with conditioned media from differentiated LPS and TNFa stimulated THP-1 macrophages. Gene expressions were analyzed by qRT$P C R$. $* P<0.05,+P<0.01, \ddagger P<0.001$ compared with control group (anakinra $0 \mathrm{ng} / \mathrm{mL}$ ) using the analysis of variance (ANOVA) with Tukey's post hoc analysis for multiple group comparison. 

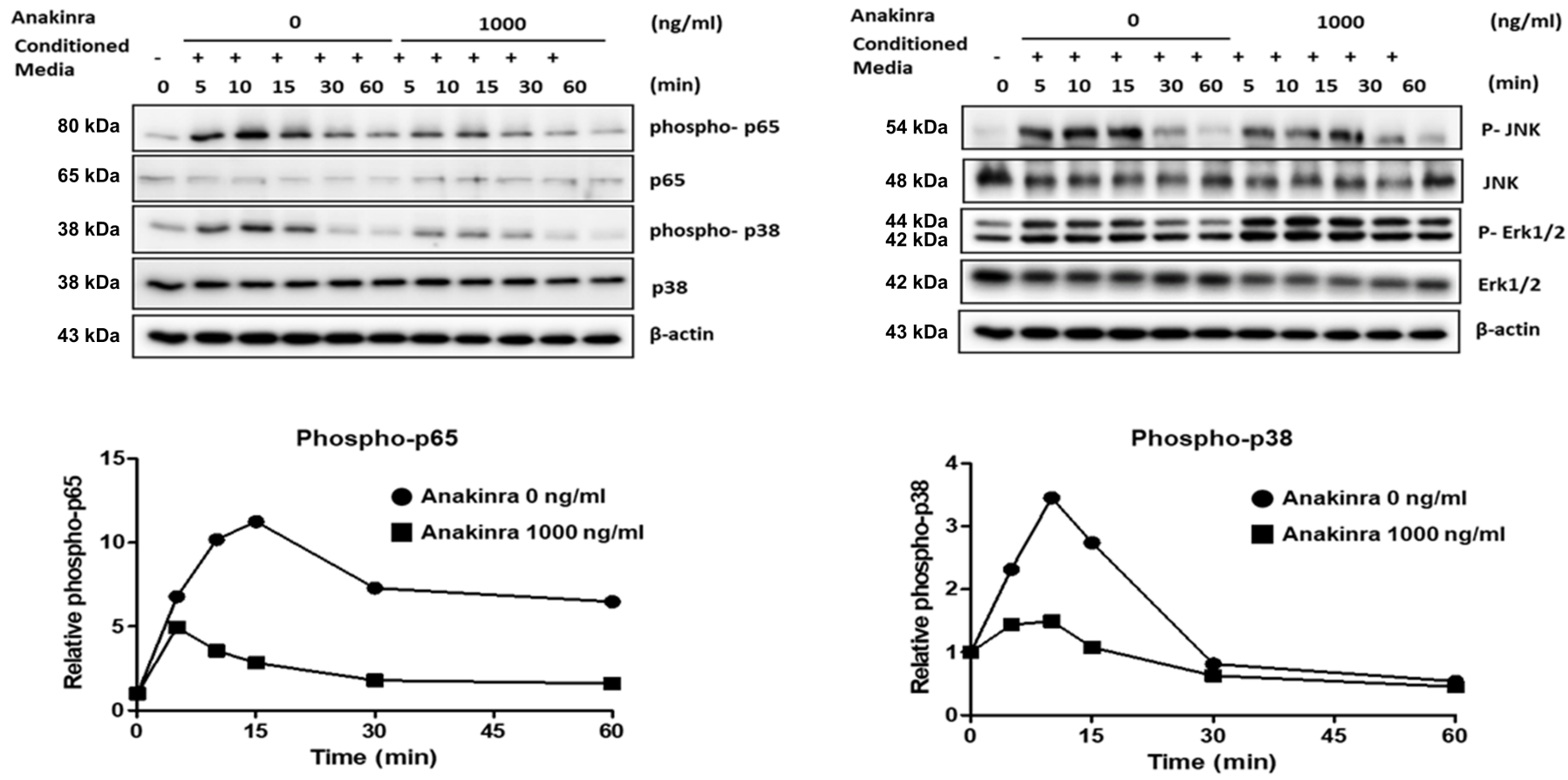

\section{Figure 4}

The effect of anakinra on the mitogen-activated protein kinase (MAPK)/nuclear factor-KB (NF-KB) pathway in RAOSMC.
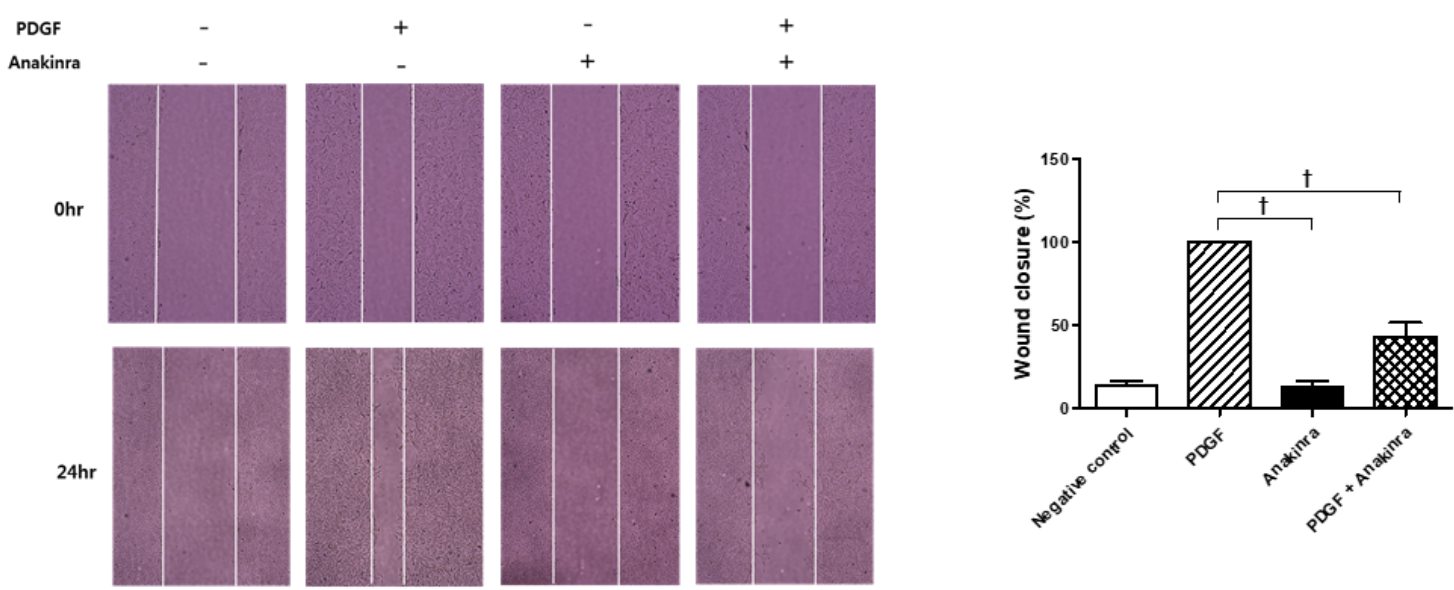


\section{Figure 5}

Wound healing assay in RAOSMC. The migration capacity was assessed by wound healing assay. Anakinra reduced cell migration in RAOSMCs compared with the positive control. $(n=3)$. Results are expressed as a percentage of PDGF-stimulated migration (mean $\pm S D$ ). $N=3$ wells, 10 fields-of-view per well. ${ }^{*} P<0.05,{ }^{*} P<0.01,+P<0.001$, versus the positive control (PDGF) group using the analysis of variance (ANOVA) with Tukey's post hoc analysis for multiple group comparison.

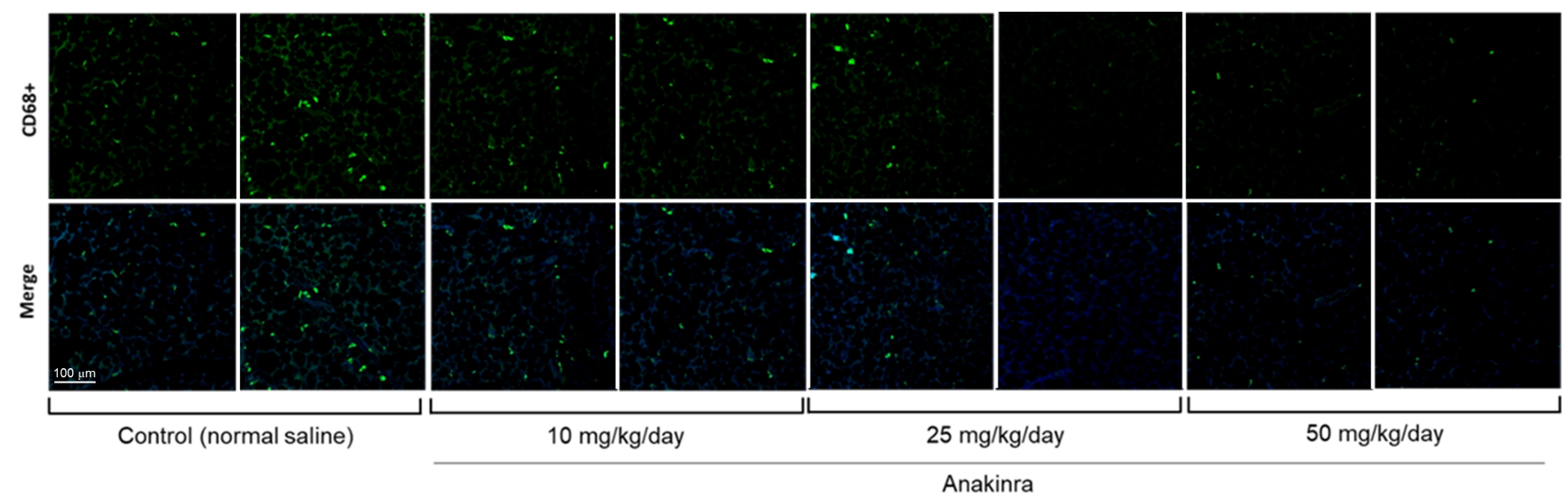

\section{Figure 6}

Immunofluorescence staining of CD68 (1:200) in visceral adipose tissue. 

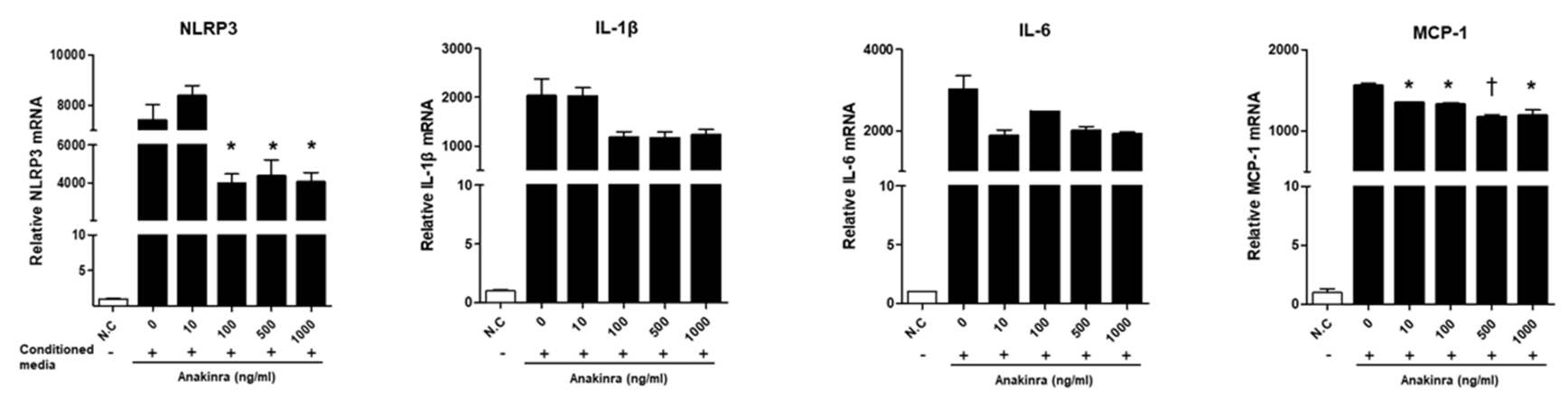

\section{Figure 7}

The effect of dose-dependent anakinra on the activated NLRP3 inflammasome and upregulated expression of inflammatory molecules in 3T3-L1 adipocytes after stimulation with conditioned media from differentiated LPS and TNFa stimulated THP-1 macrophages. Gene expressions were analyzed by qRT-PCR. * $\mathrm{P}<0.05, \mathrm{tP}<0.01, \neq \mathrm{P}<0.001$ compared with control group (anakinra $0 \mathrm{ng} / \mathrm{mL}$ ) using the analysis of variance (ANOVA) with Tukey's post hoc analysis for multiple group comparison.

\section{Supplementary Files}

This is a list of supplementary files associated with this preprint. Click to download.

- Graphicalabtract.tif

- Supplementarylnformation.pdf 